\title{
Banca extranjera y cambios en la estructura del crédito bancario en el Perú: 2001-2008 (Avances de investigación)
}

\author{
Gaby Cortez Cortez
}

\section{RESUMEN}

El ingreso de la banca extranjera al Perú, no solo ha contribuido a estimular a los bancos nacionales a través de una mayor competencia, sino a una mejora de la eficiencia, manejo de costos, y a una diversidad de productos y servicios financieros que se ofrecen. El trabajo analiza los cambios ocurridos en la estructura de los créditos directos del sistema bancario peruano, como consecuencia de la participación de bancos de origen foráneo, para lo cual se realiza una evaluación de los componentes del crédito directo tales como los créditos comerciales, de consumo, a la microempresa, e hipotecario.

Palabras clave: banca extranjera, crédito directo, estructura de crédito.

\begin{abstract}
The entry of foreign banks in Peru, has not only helped to stimulate domestic banks through greater competition, but to improved efficiency, cost management, and a variety of financial products and services that are offered. The paper analyzes the changes in the structure of direct loans from the banking system in Peru, as a result of the participation of banks of foreign origin, which conducts an assessment of the components of direct credit, such as commercial, consumer, microenterprise, and mortgage loans.
\end{abstract}

Key words: Foreign banks, direct credit, credit structure. 


\section{Gaby Cortez Cortez}

Notas Preliminares: Se considera a un banco como extranjero cuando la participación accionaría supera el 50 por ciento en manos de propietarios extranjeros.

Para los efectos del presente trabajo, los bancos con una mayor participación accionaría extranjera se señalan con un asterisco (*).

\section{INTRODUCCIÓN}

La presencia de la banca extranjera en el mercado peruano no solo ha contribuido a estimular a los bancos nacionales a través de una mayor competencia, sino también a una mayor diversidad de productos y servicios financieros.

Cuando se visualiza el negocio bancario como una aproximación de la producción se puede concebir a este negocio como una organización productiva de servicios que emplea trabajo, capital y equipos para producir activos financieros, pagos, préstamos y otros servicios.

El énfasis en la ganancia, relaciona los ingresos y gastos como inputs y outputs, de tal manera que el proceso de intermediación responde a cuan eficientemente los depósitos y otros inputs son transformados en préstamos y otros outputs.

Desde esta perspectiva, la diversidad de productos y servicios financieros puede ser una medida del estado actual del negocio bancario y una medida de la competencia y eficiencia de los bancos extranjeros y nacionales.

\section{REVISIÓN DE LA LITERATURA SOBRE EL TEMA}

Existe abundante literatura sobre la participación de la banca extranjera en diversos países del mundo, y sobre los cambios que se realizan en las economías domésticas. Por ejemplo, Hidenobu Okuda (2006) investiga los cambios en las tecnologías de producción de los bancos extranjeros y domésticos de Tailandia, en relación al aumento de la penetración de la banca extranjera mediante la estimación de las funciones de costos de 28 bancos. 


\section{Banca extranjera y cambios en la estructura del crédito bancario en el Perú}

De otro lado, el trabajo de Mark Bertus (2007) analiza la relación entre la propiedad de la banca extranjera y los tres pilares del Acuerdo de Basilea sobre el capital (regulación, supervisión y disciplina de mercado); y encuentra que en los países con gran disciplina de mercado hay una menor presencia de banca extranjera en las economías domésticas.

La investigación de Van Tassel (2007) analiza el nuevo mercado de crédito liberalizado, en donde los bancos extranjeros tienen más ventajas de costos, pero tienen menos probabilidades de tener información sobre el riesgo de crédito; mientras que los bancos domésticos que poseen tal información la guardan para futuras negociaciones.

El trabajo de Berger (2007) contrasta el comportamiento de los bancos de los países desarrollados de la "Europa Antigua" y el de la "Nueva Europa", en lo referente a la propiedad extranjera en los dos casos.

La investigación de Claessens (2001) inquiere sobre los márgenes de interés neto, los costos fijos, los impuestos, las ganancias, y la manera en que estas variables difieren entre los bancos domésticos y los extranjeros.

El trabajo de Unite y Sullivan (2003) examina la respuesta de los bancos domésticos de Filipinas a la flexibilidad de la regulación para el ingreso de bancos extranjeros que se llevó a cabo en dicho país.

\section{EL CRÉDITO BANCARIO EN EL PERÚ}

\subsection{Estructura del crédito bancario}

La estructura del crédito directo del Sistema Bancario del Perú ha mostrado modificaciones en los últimos ocho años, ya que los créditos comerciales disminuyen su participación dentro del total de créditos directos, contrayéndose desde 79.2 por ciento en el 2001 hasta 62.4 por ciento en el 2008, es decir, casi 17 puntos porcentuales menos. De otro lado, los créditos de consumo como parte de los créditos directos se incrementaron de manera importante, ya que muestran un repunte significativo al pasar del 8.6 por ciento en el 2001 hasta el 19.9 por ciento en el 2008, es decir, un incremento de 


\section{Gaby Cortez Cortez}

11.3 puntos porcentuales. También se observa que el crédito hipotecario de vivienda se expandió desde el 2001 hasta el 2005, para luego disminuir su participación dentro del total de crédito directo, pasando desde 14.8 por ciento en este último año hasta 11.9 por ciento en el 2008. Finalmente, los créditos a la microempresa sólo participan de manera residual en el total de crédito directo, pasando de 2.5 por ciento en el 2001 a 5.8 por ciento en el 2008 (Ver el Cuadro 1).

Estos cambios en la estructura de los créditos directos nos conducen a preguntarnos ¿Cuál ha sido la participación de la banca extranjera en estas modificaciones? Para tal efecto, analizaremos el comportamiento de los créditos de la banca de manera general.

Cuadro N. ${ }^{\circ}$ 1. Crédito Directo del Sistema Bancario: 2001-2008*

(En porcentajes)

\begin{tabular}{lrrrrrrrrr}
\hline & $\mathbf{2 0 0 1}$ & $\mathbf{2 0 0 2}$ & $\mathbf{2 0 0 3}$ & $\mathbf{2 0 0 4}$ & $\mathbf{2 0 0 5}$ & $\mathbf{2 0 0 6}$ & $\mathbf{2 0 0 7}$ & $\mathbf{2 0 0 8}$ & Prom. \\
\hline Comercial & 79.2 & 77.6 & 72.6 & 68.1 & 65.8 & 64.2 & 63.9 & 62.4 & 69.2 \\
A Microempresa & 2.5 & 2.3 & 2.9 & 4.3 & 5.0 & 5.3 & 5.5 & 5.8 & 4.2 \\
Consumo & 8.6 & 9.4 & 11.6 & 13.4 & 14.4 & 16.5 & 18.3 & 19.9 & 14.0 \\
Hipotecario Vivienda & 9.7 & 10.7 & 12.8 & 14.2 & 14.8 & 14 & 12.3 & 11.9 & 12.6 \\
Total & 100.0 & 100.0 & 100.0 & 100.0 & 100.0 & 100.0 & 100.0 & 100.0 & \\
\hline
\end{tabular}

*Cifras a junio

Fuente: Superintendencia de Banca y Seguros.

Elaboración: Propia

Se puede afirmar que esta modificación de la estructura de los créditos directos ha favorecido al consumo y retraído el financiamiento a la industria manufacturera, ya que en el 2002 y 2003, por ejemplo, las colocaciones a la industria manufacturera representaban el 28.02, y 28.46 por ciento de las colocaciones totales de los bancos, respectivamente, para luego producirse una declinación sostenida de estos créditos a partir del 2004, y en el 2008 constituyen tan sólo el 20.60 por ciento del total de colocaciones. En una economía en donde el mercado de capitales no es la fuente principal de financiamiento de las empresas, una disminución relativa del crédito a la industria manufactura tiene efectos no favorables para este sector y para la economía peruana (Ver el Cuadro 2). 


\section{Banca extranjera y cambios en la estructura del crédito bancario en el Perú}

Cuadro N. ${ }^{\circ}$ 2. Colocaciones de la Banca Múltiple por Sectores Económicos de Destino: 2001-2008 (En porcentajes)

\begin{tabular}{lrrrrrrrr}
\hline & $\mathbf{2 0 0 1}$ & $\mathbf{2 0 0 2}$ & $\mathbf{2 0 0 3}$ & $\mathbf{2 0 0 4}$ & $\mathbf{2 0 0 5}$ & $\mathbf{2 0 0 6}$ & $\mathbf{2 0 0 7}$ & $\mathbf{2 0 0 8}$ \\
\hline Industria Manufacturera & 22.9 & 28.02 & 28.46 & 26.21 & 23 & 21.64 & 22.1 & 20.6 \\
Comercio & 18.15 & 11.91 & 12.64 & 12.49 & 13.81 & 14.44 & 13.99 & 14.36 \\
Consumo & 8.62 & 9.41 & 11.63 & 13.43 & 14.37 & 16.46 & 18.26 & 19.97 \\
\hline
\end{tabular}

Fuente: Superintendencia de Banca y Seguros.

Elaboración: Propia

\subsection{Crédito comercial}

Si se toma en consideración la participación de los créditos comerciales / total de créditos directos otorgados por cada banco para el periodo del 2001 al 2008, se observa que en promedio el Banco de Crédito es el que más proporción de este tipo de créditos ha concedido, con 74.1 por ciento en promedio, siguiéndole el Banco Continental* con 72.7 por ciento en promedio; y Scotiabank* e Interbank con 71.1 y 60.8 por ciento, respectivamente, durante este periodo de análisis.

Sin embargo, al mismo tiempo, ocurre una disminución paulatina y sostenida de los créditos comerciales otorgados por el Banco de Crédito desde 84.3 por ciento en el 2001 a 68.3 por ciento en el 2008, es decir, una reducción de 16 puntos porcentuales; mientras que el Banco Continental* pasa de 72.3 por ciento a 73.3 por ciento del 2001 al 2008, es decir, no solo mantiene la participación de sus recursos dirigidos a este tipo de créditos, sino que se incrementa ligeramente, de tal forma que parece haber logrado un nivel con el que se encuentra operando satisfactoriamente.

De otro lado, Scotiabank* cede su posición al Banco Continental* a partir del 2005; e Interbank indica una disminución desde el 79.2 por ciento hasta el 49.3 por ciento del 2001 al 2008, es decir,casi 30 puntos porcentuales menos (Ver el Cuadro 3).

Se puede señalar entonces que es clara la disminución de los créditos comerciales como parte de los créditos directos totales otorgados por los bancos. 


\section{Gaby Cortez Cortez}

Cuadro N. ${ }^{\circ}$ 3. Crédito Comercial / Crédito Directo por Empresa Bancario: 2001-2008 (En porcentajes)

\begin{tabular}{lrrrrrrrrr}
\hline & $\mathbf{2 0 0 1}$ & $\mathbf{2 0 0 2}$ & $\mathbf{2 0 0 3}$ & $\mathbf{2 0 0 4}$ & $\mathbf{2 0 0 5}$ & $\mathbf{2 0 0 6}$ & $\mathbf{2 0 0 7}$ & $\mathbf{2 0 0 8}$ & Prom. \\
\hline B. Continental $^{*}$ & 72.3 & 75.3 & 70.6 & 70.5 & 72.0 & 73.3 & 73.9 & 73.3 & 72.7 \\
B. de Crédito* $^{\text {Scotiabank* }}$ & 84.3 & 82.1 & 77.7 & 71.9 & 69.9 & 69.1 & 69.2 & 68.3 & 74.1 \\
Interbank $_{\text {Total Banca Múltiple }}$ & 81.8 & 81.1 & 73.1 & 70.2 & 68.5 & 64.0 & 64.6 & 65.4 & 71.1 \\
\hline
\end{tabular}

*Cifras a junio

Fuente: Superintendencia de Banca y Seguros.

Elaboración: Propia

\subsection{Crédito de Consumo}

El crédito de consumo ha tenido un aumento importante en la economía peruana, tal como se observa en los cuadros 1 y 2 , pasando de un 8.6 por ciento del total de créditos directos otorgados por la banca en el 2001, a un 19.9 por ciento en junio de 2008. De esta forma, se movilizó recursos financieros a este segmento del orden de 3,156,085 mil nuevos soles en el 2001, y de 15, 221,256 mil nuevos soles en el 2008; es decir, casi se ha quintuplicado los recursos dirigidos a este sector.

Para medir la participación del crédito de consumo dentro de la estructura de créditos directos por cada banco, se emplea el ratio crédito de consumo / total de créditos directos otorgados por dicho banco. Es así que se encuentra que en términos generales, casi todos los bancos se mueven hacia un aumento en la participación de tales créditos de consumo, pero en distinto grado. De esta forma, dentro de los bancos grandes destaca la participación de Interbank y de Scotiabank* en ese orden, siguiendo los Bancos Continental* y de Crédito luego. En cuanto a los bancos medianos y pequeños, la participación en el otorgamiento de los créditos de consumo es mayor tal como puede verse en el Cuadro 4.

De otro lado, cuando se analiza la estructura de los créditos de consumo del total de bancos, es importante notar que durante el periodo de análisis en términos promedio, el Banco de Crédito es el que más créditos de consumo otorgó (19.2 por ciento); siguiéndole, en segundo lugar, el Banco Wiese Sudameris* (Scotiabank) conjuntamente con Interbank (18.7 por cien- 


\section{Banca extranjera y cambios en la estructura del crédito bancario en el Perú}

to), mientras que el Banco Continental se sitúa en tercer lugar (15.0 por ciento). Ver Cuadro 4.1. Estos hechos nos muestran la disminución del liderazgo en este nicho por parte de Scotiabank*, así como el repunte de Interbank. Asimismo, se debe hacer notar que algunos bancos pequeños han hecho su aparición en el mercado bancario tales como los Bancos Falabella* y Ripley*, que en el 2008 participan del 6.8 y 5.9 por ciento de los créditos de consumo.

Cuadro N. ${ }^{\circ}$ 4. Crédito de Consumo / Crédito Directo por Empresa Bancaria:2001-2008*

(En porcentajes)

\begin{tabular}{lrrrrrrrrr}
\hline \multicolumn{1}{c}{ Banco } & $\mathbf{2 0 0 1}$ & $\mathbf{2 0 0 2}$ & $\mathbf{2 0 0 3}$ & $\mathbf{2 0 0 4}$ & $\mathbf{2 0 0 5}$ & $\mathbf{2 0 0 6}$ & $\mathbf{2 0 0 7}$ & $\mathbf{2 0 0 8}$ & Prom. \\
\hline B. Continental* & 7,7 & 7,6 & 9,6 & 10,0 & 9,5 & 10,3 & 12 & 12,8 & 9,9 \\
B. de Crédito & 6,6 & 5,6 & 6,5 & 7,5 & 7,9 & 9,3 & 10,8 & 11,7 & 8,2 \\
Scotiabank* & 9,6 & 12,1 & 14,7 & 16,8 & 17,0 & 18,9 & 19,1 & 19,0 & 15,9 \\
Interbank & 15,2 & 19,9 & 24,9 & 28,7 & 31,7 & 36,8 & 34,7 & 36,4 & 28,5 \\
Citibank* & 10,8 & 11,7 & 12,8 & 16,3 & 22,9 & 36,3 & 40,8 & 45,8 & 24,7 \\
B. del Trabajo* & 57,2 & 48,9 & 46,4 & 44,1 & 41,9 & 43,8 & 48,8 & 45,4 & 47,1 \\
B. Financiero* & 4,3 & 9,1 & 14,5 & 17,9 & 17,2 & 22,2 & 26,9 & 27,6 & 17,5 \\
B. de Comercio & 21,6 & 24,6 & 31,7 & 53,6 & 67,1 & 64 & 66,8 & 70,7 & 50,0 \\
Total Banca Múltiple & 8,6 & 9,4 & 11,6 & 13,4 & 14,4 & 16,5 & 18,3 & 19,9 & \\
\hline
\end{tabular}

Fuente: Superintendencia de Banca y Seguros.

Elaboración: Propia *A Junio 2008.

Cuadro N. ${ }^{\circ}$ 4.1. Crédito de Consumo/ Total Crédito Consumo de Bancos: 2001-2008 (En porcentajes)

\begin{tabular}{lrrrrrrrrr}
\hline & $\mathbf{2 0 0 1}$ & $\mathbf{2 0 0 2}$ & $\mathbf{2 0 0 3}$ & $\mathbf{2 0 0 4}$ & $\mathbf{2 0 0 5}$ & $\mathbf{2 0 0 6}$ & $\mathbf{2 0 0 7}$ & $\mathbf{2 0 0 8}$ & Prom. \\
\hline B.Crédito & 20,8 & 17,5 & 19,1 & 18,3 & 18,7 & 19,0 & 20,5 & 19,7 & 19,2 \\
Scotiabank* & 24,1 & 22,3 & 18,7 & 18,1 & 16,9 & 18,2 & 15,8 & 15,3 & 18,7 \\
B.Continental* & 13,7 & 13,2 & 14,9 & 14,9 & 14,5 & 16,1 & 16,7 & 15,6 & 15,0 \\
Interbank & 13,3 & 18 & 20,6 & 21,5 & 20,9 & 19,6 & 17,8 & 17,7 & 18,7 \\
Citibank* & 6,3 & 5,5 & 4,4 & 4,5 & 6,2 & 8,7 & 6,6 & 5,3 & 5,9 \\
B.Falabella* & & & & & & & 7,5 & 6,8 & 7,2 \\
B.Ripley* & & & & & & & & 5,9 & 5,9 \\
Sub total & 78,2 & 76,5 & 77,7 & 77,3 & 77,2 & 81,6 & 84,9 & 86,3 & \\
\hline
\end{tabular}

Fuente: Superintendencia de Banca y Seguros.

Elaboración: Propia $\quad *$ *A Junio 2008. 


\section{Gaby Cortez Cortez}

Asimismo, consideramos importante revisar el comportamiento del costo del crédito de consumo para los clientes de los bancos, en la medida que se asume que con la presencia de bancos extranjeros, la mayor competencia conduciría a mejores condiciones de costo del crédito para los usuarios finales del mismo. Es así que se tiene que las tasas de interés máximas para el crédito de consumo en moneda nacional, para un préstamo de S/. 5,000 nuevos soles a 12 meses de plazo por ejemplo, muestra un aumento en los casos de los bancos de Crédito, Scotiabank*, Citibank* y del Trabajo* (ahora forma parte del grupo Scotiabank), mientras que Mibanco ha disminuido su tasa de interés máxima. Ver el Cuadro 4.2. Al mismo tiempo, puede observarse que a pesar que Interbank tiene una tasa de interés mayor al promedio del sistema, los volúmenes de préstamos que otorga se han incrementado significativamente -debido al direccionamiento de los créditos de consumo masivo a través de Plaza Vea- a diferencia de los bancos pequeños como el Interamericano de Finanzas*, Financiero* y de Comercio, que a pesar de tener tasas menores que el promedio, no movilizan mayores recursos a este tipo de crédito.

En cuanto a las tasas de interés máximas de los créditos de consumo en dólares; por ejemplo, para un préstamo de US\$1,500 a 12 meses de plazo, se encuentra que las tasas máximas de siete de once bancos del sistema se han incrementado, y en algunos casos de manera impresionante. Los casos de incremento de tasas de interés máximas sustanciales son las de los Bancos del Trabajo* (29.88 puntos porcentuales de aumento), Banco Interamericano de Finanzas* (10.88 puntos porcentuales de aumento), Banco Continental* (9.94 puntos porcentuales de incremento), y Banco de Crédito (5.6. puntos porcentuales de incremento). Los otros bancos han aumentado las tasas máximas en menor magnitud.

Esta elevación de las tasas de interés máximas nos indicaría que ante la fuerte demanda por este tipo de créditos los bancos hacen uso de su poder de mercado que poseen para poner sus propias condiciones, mostrándonos ade-

más la disminución de la competencia entre los bancos, y la consolidación de segmentos de mercado. 


\section{Banca extranjera y cambios en la estructura del crédito bancario en el Perú}

Cuadro N. ${ }^{\circ}$ 4.2. Tasas de Interés Bancaria de Crédito de Consumo

\begin{tabular}{lrrrrrrrr}
\hline \multirow{1}{*}{ Bancos } & \multicolumn{3}{c}{ S/.5,000 a 12 meses } & \multicolumn{3}{c}{ US\$ 1,500 a 12 meses } \\
& \multicolumn{2}{c}{ TCE Mínima } & \multicolumn{2}{c}{ TCE Máxima } & \multicolumn{2}{c}{ TCE Mínima } & \multicolumn{1}{c}{ TCE Máxima } \\
& 31.08 .07 & 19.09 .08 & 31.08 .07 & 19.09 .08 & 31.08 .07 & 19.09 .08 & 31.08 .07 & 19.09 .08 \\
\hline B. Interameric.* & - & - & 21,13 & 21,22 & - & - & 23,70 & 34,18 \\
B. Financiero* & 16,03 & 16,03 & 24,17 & 24,17 & 16,03 & 16,03 & 24,18 & 24,18 \\
Citibank * & 22,36 & 24,31 & 25,40 & 34,56 & 18,46 & 20,54 & 22,52 & 24,56 \\
B.Comercio & - & - & 29,59 & 29,59 & - & - & 23,78 & 23,78 \\
B. de Credito & 18,69 & 22,19 & 39,17 & 41,87 & 18,71 & 25,11 & 31,99 & 37,59 \\
Fin. Cordillera* & 22,99 & 22,99 & 42,37 & 42,37 & 21,85 & 21,85 & 41,87 & 41,87 \\
B. Continental* & - & - & 42,83 & 42,83 & - & - & 36,83 & 46,7652 \\
B. del Trabajo* & 40,75 & 41,68 & 49,72 & 55,99 & 29,46 & 39,65 & 38,93 & 68,81 \\
Interbank & 36,61 & 36,61 & 52,40 & 52,40 & 26,26 & 26,26 & 43,55 & 43,55 \\
Scotiabank * & 31,64 & 26,99 & 54,33 & 55,14 & 34,08 & 26,39 & 50,81 & 51,45 \\
Mibanco & 57,10 & 57,10 & 62,15 & 56,29 & - & - & 41,95 & 44,06 \\
Promedio & 30,77 & 30,99 & 40,30 & 41,49 & 23,55 & 25,12 & 38,01 & 40,07 \\
\hline
\end{tabular}

Fuente: Superintendencia de Banca y Seguros

Elaboracion: Propia

\subsection{Crédito a microempresas}

En lo concerniente a la participación, de los créditos a la microempresa/total de créditos directos de cada banco, se observa que los bancos pequeños, tales como Mibanco y el Banco del Trabajo*, canalizan de manera significativa sus recursos hacia este tipo de crédito, en un 70.7 y 42.7 por ciento en promedio, respectivamente. Mientras que los bancos grandes como los bancos Crédito y Scotiabank* empiezan a incrementar la dirección de recursos a este tipo de créditos, sin llegar a ser parte importante del total de sus colocaciones, ya que en promedio constituyen el 4 por ciento del total de sus préstamos (Ver el Cuadro 5). Por lo tanto, a pesar del aumento en niveles absolutos de estos créditos, este sector del mercado no ha logrado aun ser una parte significativa dentro del total de créditos otorgados por los bancos. 


\section{Gaby Cortez Cortez}

Cuadro N. ${ }^{\circ}$ 5. Crédito A la Microempresa / Crédito Directo por Empresa Bancaria: 2001-2008 ( En porcentajes)

\begin{tabular}{lrrrrrrrrr}
\hline & $\mathbf{2 0 0 1}$ & $\mathbf{2 0 0 2}$ & $\mathbf{2 0 0 3}$ & $\mathbf{2 0 0 4}$ & $\mathbf{2 0 0 5}$ & $\mathbf{2 0 0 6}$ & $\mathbf{2 0 0 7}$ & $\mathbf{2 0 0 8 *}$ & Prom. \\
\hline Mibanco & 75,9 & 66,8 & 68,8 & 69,5 & 70,4 & 69,9 & 71,6 & 72,3 & 70,7 \\
B. del Trabajo* & 33,6 & 42,9 & 42,9 & 41,9 & 40,2 & 38,1 & 49,4 & 52,6 & 42,7 \\
Scotiabank * & 1,4 & 1,9 & 2,5 & 3,2 & 4,5 & 5,6 & 6,3 & 6,6 & 4,0 \\
B. de Crédito & 0,8 & 1,3 & 1,8 & 4,7 & 5,2 & 5,8 & 5,8 & 5,7 & 3,9 \\
Total Banca Múltiple & 2,5 & 2,3 & 2,9 & 4,3 & 5,0 & 5,3 & 5,5 & 5,8 & \\
\hline
\end{tabular}

* Cifras a junio. Fuente: Superintendencia de Banca y Seguros. Elaboración: Propia

De otro lado, cuando se revisa la estructura de los créditos a la microempresa del total de bancos, encontramos importante mencionar que el crédito a la microempresa ha mostrado cambios en el comportamiento de sus actores en los ocho últimos años, de tal manera que, por ejemplo, en el 2001, el Banco Continental* colocaba el 43.6 por ciento del total de tales créditos en el sistema; Mibanco, el 17.3 por ciento; el Banco del Trabajo*, el 15.0 por ciento; el Banco Wiese Sudameris* (Scotiabank), el 12.3 por ciento; y el Banco de Crédito tan sólo el 8.6 por ciento. Mientras que en junio de 2008, el primer lugar lo ocupa el Banco de Crédito con un 33.5 por ciento de los créditos totales a las Microempresas; le sigue Mibanco, en segundo lugar, con el 30.1 por ciento; Scotiabank*, con el 18.3 por ciento; y el Banco del Trabajo*, con el 13.4 por ciento. De estas cifras, se observa la retirada del Banco Continental* de este nicho; el ingreso del Banco de Crédito en primer lugar, ocupando Mibanco el segundo lugar, así como la mejora de Scotiabank* en este nuevo espacio. Entonces, este nicho ya no es más de los bancos chicos, sino también de los bancos grandes.

Cuadro N. ${ }^{\circ}$ 5.1. Crédito A Microempresa / Total Crédito a Microempresa :2001-2008 (En porcentajes)

\begin{tabular}{lrrrrrrrrr}
\hline & $\mathbf{2 0 0 1}$ & $\mathbf{2 0 0 2}$ & $\mathbf{2 0 0 3}$ & $\mathbf{2 0 0 4}$ & $\mathbf{2 0 0 5}$ & $\mathbf{2 0 0 6}$ & $\mathbf{2 0 0 7}$ & $\mathbf{2 0 0 8}$ & Prom. \\
\hline B. Continental* & 43,6 & 13,1 & 7,9 & 3,7 & 2,5 & 1,6 & 1,2 & 1,0 & 9,3 \\
B.Crédito & 8,6 & 16,8 & 21,9 & 36,2 & 35,7 & 1,0 & 36,1 & 33,5 & 23,7 \\
Mibanco & 17,3 & 25,1 & 26,4 & 19,0 & 23,0 & 26,7 & 29,0 & 30,1 & 24,6 \\
Scotiabank* & 12,3 & 14,1 & 12,6 & 10,8 & 11,6 & 16,7 & 17,2 & 18,3 & 14,2 \\
B.Trabajo* & 15,0 & 29,1 & 30,3 & 24,7 & 20,0 & 15,8 & 13,8 & 13,4 & 20,3 \\
Sub total & 96,8 & 98,2 & 99,1 & 94,4 & 92,8 & 61,8 & 97,3 & 96,3 & \\
\hline
\end{tabular}

Fuente: Superintendencia de Banca y Seguros. Elaboración: Propia. Elaboracion: Propia 


\section{Banca extranjera y cambios en la estructura del crédito bancario en el Perú}

También, es necesario indicar que en el 2001, el Banco Continental canalizó 404,022 mil nuevos soles en préstamos a las microempresas; Mibanco, 160,472 mil nuevos soles; el Banco del Trabajo*, 139,016 mil nuevos soles; y el Banco Wiese Sudameris*, 113,660 mil nuevos soles. En junio de 2008, el Banco de Crédito otorgó 1,475,749 mil nuevos soles y Mibanco 1,325,144 mil nuevos soles. El total de recursos dirigidos a estos préstamos fue de 927,136 mil nuevos soles en el 2001 y de 4,404,282 mil nuevos soles al 30 de junio de 2008, es decir, los recursos dirigidos a estos créditos se incrementaron en 4.7 veces.

Por otro lado, en cuanto al costo de los préstamos, las tasas de interés que se cobran por este tipo de crédito, en el caso específico de préstamos para capital de trabajo de US\$ 3000 a 9 meses, por ejemplo, se han visto incrementadas, desde un promedio de 40.30 por ciento en agosto de 2007 a 43.51 por ciento, al 19 de setiembre de 2008 (Ver Cuadro 5.2). Es importante señalar que los Bancos del Trabajo y Mibanco aumentan las tasas máximas de interés que cobran por este tipo de producto en 20.58 puntos y 2.12 puntos, respectivamente. Ambas tasas se sitúan por encima de la tasa promedio del sistema para este tipo de préstamos. Esta diferencia de tasas favorece de manera especial al Banco de Crédito, el que con una menor tasa, así como con toda una política crediticia dirigida a este sector creciente de clientes ha colocado un mayor volumen de recursos en este segmento, que antes era un espacio de algunos bancos pequeños. El caso contrario es el del Banco de Comercio, por ejemplo, que a pesar de ofrecer una tasa de interés mucho menor que el promedio del sistema, no logra tener una mayor significación en el otorgamiento de estos créditos, lo que nos indica que no solo entra a tallar como elemento importante el costo del crédito, sino otros factores adicionales propios de cada banco.

Cuadro N. ${ }^{\circ}$ 5.2. Tasas de Interés por Capital de Trabajo

\begin{tabular}{lrrrr}
\hline \multicolumn{1}{c}{ Bancos } & \multicolumn{4}{c}{ A Microempresas US\$3,000 a 9 meses } \\
& \multicolumn{2}{c}{ TCE Mínima } & \multicolumn{2}{c}{ TCE Máxima } \\
\hline B.Comercio & 31.08 .07 & 19.09 .08 & 31.08 .07 & 19.09 .08 \\
B.Interameric.* & - & - & 26,69 & 26,69 \\
B.Trabajo* & 29,79 & - & 30,56 & 30,56 \\
Scotiabank * & - & 37,27 & 38,64 & 59,22 \\
Mibanco & 36,90 & 37,11 & 39,61 & 39,32 \\
B. Crédito & - & - & 41,95 & 44,07 \\
B. Continental* & - & - & 62,58 & 42,15 \\
Promedio & & & 40,30 & 62,58 \\
\hline
\end{tabular}

Fuente: Superintendencia de Banca y Seguros Elaboracion: Propia 


\section{Gaby Cortez Cortez}

\section{5. Crédito hipotecario de vivienda}

Con el ratio crédito hipotecario de vivienda de cada banco/total de créditos directos de dicho banco, se muestra una expansión, especialmente del 2001 al 2005, para luego empezar a disminuir como porcentaje del total de créditos bancarios del 2006 al 2008. El Banco Continental ha otorgado el mayor porcentaje de estos créditos en promedio del 2001 al 2008 con un 15.9 por ciento en promedio; siguiéndole el Banco de Crédito, con un 13.8 por ciento en promedio; e Interbank y Scotiabank*, con 10.4 y 9.1 por ciento, respectivamente (Ver el Cuadro 6).

La estructura de los créditos hipotecarios de vivienda del total de bancos nos indica que también se han producido cambios en sus ofertantes durante el periodo de análisis, ya que en el 2001, el Banco de Crédito otorgaba el 23.3 por ciento de los mismos, el Continental*, el 20.2 por ciento; el Santander Central Hispano* (absorbido por el Banco de Crédito), el 18.5 por ciento; y el Wiese Sudameris* (Scotiabank), el 16.0 por ciento. En el 2008 (junio), se tiene que el Banco de Crédito participa del 40.4 del total de estos créditos, le sigue el Continental* con el 27.9 por ciento; Scotiabank*, con 12.1 por ciento; e Interbank, con el 10.8 por ciento. Es notoria la participación importante de los Bancos de Crédito y Continental* en este rubro, ya que en conjunto, explican el 68.3 por ciento de estos préstamos en lo que va del presente año.

Cuadro N. ${ }^{\circ}$ 6. Créditos Hipotecarios / Crédito Directo por Empresa Bancaria: 2001-2008 (En \%)

\begin{tabular}{lrrrrrrrrr}
\hline \multicolumn{1}{c}{ Banco } & $\mathbf{2 0 0 1}$ & $\mathbf{2 0 0 2}$ & $\mathbf{2 0 0 3}$ & $\mathbf{2 0 0 4}$ & $\mathbf{2 0 0 5}$ & $\mathbf{2 0 0 6}$ & $\mathbf{2 0 0 7}$ & $\mathbf{2 0 0 8}$ & Prom. \\
\hline B. Continental & 12,8 & 15,3 & 18,6 & 18,8 & 18,0 & 16 & 13,9 & 13,6 & 15,9 \\
B. de Crédito & 8,3 & 11,0 & 14,0 & 15,9 & 17,0 & 15,8 & 14,2 & 14,3 & 13,8 \\
Scotiabank * & 7,2 & 5,0 & 9,8 & 9,9 & 10,4 & 11,6 & 10,1 & 9,0 & 9,1 \\
Interbank & 5,5 & 6,8 & 9,2 & 10,5 & 12,0 & 12,6 & 13,4 & 13,2 & 10,4 \\
Total de Banca & 9,7 & 10,7 & 12,8 & 14,2 & 14,8 & 14 & 12,3 & 11,9 & 12,6 \\
Múltiple & & & & & & & & &
\end{tabular}

Fuente: Superintendencia de Banca y Seguros

Elaboracion: Propia. * A Junio 2008.

En lo referente a las tasas de interés mínimas de los créditos hipotecarios para vivienda para un crédito de US\$ 40,000 a 15 años, estos han disminuido al 19 de 


\section{Banca extranjera y cambios en la estructura del crédito bancario en el Perú}

septiembre del 2008 respecto a las que estaban vigentes al 31 de agosto del 2007, con excepción de las del Banco de Crédito. Asimismo, las tasas de interés máximas para este tipo de crédito no se han incrementado. Para un préstamo de US\$80 000 a 15 años, las tasas máximas han disminuido en promedio, con excepción de las de los Bancos de Crédito e Interamericano de Finanzas* (Ver el Cuadro 6.1). Esta disminución de las tasas de interés es una forma de atraer una mayor demanda por este tipo de préstamos.

Cuadro $\mathbf{N}^{\circ}$ 6.1. Tasas de Interés de Crédito Hipotecario para Vivienda

\begin{tabular}{llllll}
\hline \multirow{1}{*}{\multicolumn{1}{c}{ Bancos }} & \multicolumn{3}{c}{ US\$ 40,000 15 años } & \multicolumn{2}{c}{ US\$ 80,000 15 años } \\
& 31.08 .07 & 19.09 .08 & 19.09 .08 & 31.08 .07 & 19.09 .08 \\
\hline B.Financiero* & 11,45 & - & - & 11,13 & - \\
B.Credito & 12,16 & 13,16 & 13,42 & 12,10 & 13,11 \\
Scotiabank * & 12,19 & 10,61 & 11,12 & 12,11 & 10,55 \\
B.Interamericano* & 12,40 & 11,06 & 13,59 & 12,17 & 13,53 \\
B. Falabella* & 12,47 & 11,28 & 11,98 & 12,33 & 11,98 \\
Interbank & 12,76 & 11,32 & 12,82 & 12,47 & 12,19 \\
B.Continental* & 13,99 & & 13,9855 & 12,94 & 12,94 \\
Mibanco & 19,99 & & 17,19 & 19,99 & 17,19 \\
HSBC* & & 10,47 & 11,71 & & 11,64 \\
Promedio & 13,43 & 11,32 & 13,23 & 13,16 & 12,89 \\
\hline
\end{tabular}

Fuente: Superintendencia de Banca y Seguros

Elaboracion: Propia

\section{CONCLUSIONES PRELIMINARES}

El sector bancario peruano se caracteriza por una diferenciación en sus productos y servicios financieros, a pesar que su negocio principal es moderadamente homogéneo. Esta diferenciación de productos ha establecido clientes o nichos de mercado, lo que ha permitido a los bancos, principalmente a los más grandes, sean estos nacionales o extranjeros, disfrutar del poder de mercado sobre estos nichos, para lo cual han desarrollado estrategias mediante las cuales diferencian el producto de un banco de los 


\section{Gaby Cortez Cortez}

productos de sus competidores en el diseño, servicios relacionados, calidad, ubicación y otros atributos:

- La estructura de los créditos de los bancos se ha visto modificada en los ocho últimos años, disminuyendo la participación de los créditos comerciales como parte de los créditos directos totales de los bancos, desde 79.2 por ciento en el 2001 hasta 62.4 por ciento a junio de 2008. El Banco de Crédito reduce su participación en los créditos comerciales desde 84.3 por ciento en el 2001 a 68.3 por ciento en el 2008; mientras que el Banco Continental*, prácticamente, se mantiene en 73.3 por ciento en el 2008 en el otorgamiento de créditos directos. De otro lado, los créditos de consumo que otorgan los bancos han aumentado de manera notable su participación en el total de créditos directos de los mismos, pasando de 9.7 por ciento en el 2001 a 19.9 por ciento a junio de 2008 .

- Se puede afirmar que esta modificación de la estructura relativa de los créditos directos ha favorecido al consumo y retraído el financiamiento a la industria manufacturera, lo que se ve corroborado por la reducción de las colocaciones totales de los bancos a este sector económico desde 28.0 por ciento en el 2002, 2003 hasta 20.6 por ciento a junio del 2008. En una economía en donde el mercado de capitales no es la fuente principal de financiamiento de las empresas, una disminución relativa del crédito a la industria manufacturera tiene efectos no favorables para este sector.

- Las tasas de interés de los créditos de consumo se han visto incrementadas en términos generales, tanto en moneda nacional como en moneda extranjera, reflejando la capacidad que tienen los bancos con poder de mercado de establecer condiciones más costosas para el acceso al financiamiento de las familias.

- Los créditos a la microempresa han mostrado cambios en el comportamiento de sus actores en el periodo de análisis, de tal forma que en el 2008 el Banco de Crédito es el principal otorgante de estos recursos, siguiéndole Mibanco, Scotiabank*, y el Banco del Trabajo* en ese orden, por lo que se puede indicar que este nicho al ser rentable permite que participen los bancos grandes y pequeños simultáneamente. 


\section{Banca extranjera y cambios en la estructura del crédito bancario en el Perú}

\section{REFERENCIAS BIBLIOGRÁFICAS}

Berger, A (2007). Obstacles to a global banking system: "Old Europe" vs. "New Europe". Journal of Banking \& Finance. 31: 1955-1973.

Bertus, M, Jahera, J (2008). A note on foreign ownership and monitoring: An international comparison. Journal of Banking \& Finance. 32: 338-345.

Okuda, H, Rungsomboon,S (2006). Comparative cost study of foreign and Thai domestic banks in 1990-2002: Its policy implications for a desirable banking industry structure. Journal of Asian Economics. 17: 714-737.

Unite, A, Sullivan, M (2003). The effect of foreign entry and ownership structure on the Philippine domestic banking market. Journal of Banking \& Finance. 27: 2323-2345.

Van Tassel, E, Vishwasrao, S (2007). Asymmetric Information and the mode of entry in foreign credit markets. Journal of Banking \& Finance. 31: 3742-3760. 